\title{
Laïcité au Mexique et en Amérique latine
}

Comparaisons

\section{Roberto Blancarte}

\section{(2) OpenEdition}

Journals

Édition électronique

URL : http://journals.openedition.org/assr/21205

DOI : $10.4000 /$ assr.21205

ISSN : $1777-5825$

Éditeur

Éditions de l'EHESS

Édition imprimée

Date de publication : 1 juin 2009

Pagination : 17-40

ISBN : 978-2-7132-2216-0

ISSN : 0335-5985

Référence électronique

Roberto Blancarte, "Laïcité au Mexique et en Amérique latine », Archives de sciences sociales des religions [En ligne], 146 | avril-juin 2009, mis en ligne le 01 juillet 2012, consulté le 30 avril 2019. URL : http://journals.openedition.org/assr/21205; DOI : 10.4000/assr.21205 


\section{Roberto Blancarte}

\section{Laïcité au Mexique et en Amérique latine Comparaisons}

Le Mexique est peut-être le pays le plus laïque de l'Amérique latine. Il partage avec l'Uruguay et Cuba une laïcité en pleine progression, même si celle-ci y prend parfois les formes d'un certain laïcisme (Blancarte, 2008a) ${ }^{1}$. Cependant, la laïcité mexicaine, de par son histoire et son parcours idéologique, est particulière. Suitelle un modèle spécifique ? Sert-elle de modèle à d'autres pays d'Amérique? En quoi le modèle mexicain de laïcité est-il différent de celui des autres modèles latino-américains? Telles sont les questions auxquelles cet article souhaiterait répondre. Pour ce faire, je propose une analyse théorique et un aperçu historique de la laïcité telle qu'elle s'est développée au Mexique et en Amérique latine. Je chercherai dans un second temps à comparer ces expériences latino-américaines à d'autres expériences laïques dans le monde - pays occidentaux et semioccidentalisés -, afin d'approfondir le phénomène de la laicité en général, audelà de ses spécificités latino-américaines. J'ai déjà précisé dans un texte antérieur (2007) que mon approche théorique et méthodologique cherchait à construire un système de «miroirs culturels » qui pourrait débloquer un débat souvent confus et ethnocentrique autour de l'ampleur, la portée, les limites et l'avenir de la laïcité.

\section{Définir la laïcité}

Il y a quelques années, j'ai défini la laïcité comme "un régime social de coexistence harmonieuse, dont les institutions politiques sont principalement légitimées par la souveraineté populaire et non par des éléments religieux » (2000). J'expliquais ainsi que, à un certain moment de l'histoire de l'Occident, le pouvoir politique avait cessé d'être légitimé par le sacré et la souveraineté n'était plus incarnée par une seule personne (le monarque). Au cours de ce processus, les monarchies cessèrent d'être absolues et devinrent constitutionnelles. Les

1. J'entends par laïcité un état de choses, un régime social particulier, tandis que le laïcisme est plutôt une attitude de combat pour instaurer ce régime. Voir mon article "Laicidad y laicismo en América latina », 2008a. 
États-Unis, la France ou le Mexique, et en général les pays de l'Amérique latine, connurent l'instauration de républiques. Les rois devinrent pratiquement des figures décoratives ou disparurent, la souveraineté fut transmise au peuple, raison pour laquelle la démocratie représentative et la laïcité sont intrinsèquement liées.

Cette définition, centrée sur l'idée du passage de la légitimité octroyée par le sacré à une forme d'autorité émanant du peuple, nous permet de comprendre que la laïcité, comme la démocratie, est plus un procès qu'une forme fixe ou achevée. De même, si l'on ne peut affirmer l'existence d'une société absolument démocratique, il n'existe pas, dans la réalité, de système politique qui soit complètement et définitivement laïque. Il subsiste très souvent des formes de sacralisation du pouvoir, même sous des schémas qui ne sont pas strictement religieux. Nous savons par exemple, depuis Durkheim, que de nombreuses cérémonies civiques ne sont, au fond, que des rituels de substitution pour regrouper la société sous de nouvelles valeurs communes. D'où la proposition aujourd'hui, chez certains, d'une laïcisation de la laïcité pour dépasser sa sacralité (Haarscher, 1998 : 121). Mais ceci explique également que certaines sociétés formellement laiques ou qui vivent sous un régime de séparation sont encore soumises à une forte influence sociale et politique des institutions religieuses. C'est le cas de nombreux pays latino-américains, où la hiérarchie catholique fait sentir le poids de son influence dans la mise en place de nombreuses politiques publiques.

Définir la laicité comme un processus de transition des formes de légitimité sacrées vers des formes démocratiques ou fondées sur la volonté populaire nous permet également de comprendre que la laiicité n'est pas exactement la même chose que la séparation des Églises et de l'État. De fait, il existe de nombreux États qui ne sont pas formellement laiques, mais qui élaborent des politiques publiques étrangères à la normativité doctrinale des Églises et qui font reposer leur légitimité sur la souveraineté populaire plus que sur toute autre forme de consécration ecclésiastique. Des pays comme le Danemark ou la Norvège, qui ont des Églises nationales - par exemple l’Église luthérienne dont les ministres du culte sont considérés comme fonctionnaires -, sont toutefois laïques dans la mesure où leurs formes de légitimation politique sont essentiellement démocratiques et qu'ils adoptent des politiques publiques étrangères à la propre morale de l'Église officielle.

Le critère de séparation entre les affaires de l'État et celles de l'Église est souvent confondu avec celui de laïcité, parce que les États laïques ont adopté dans la pratique des mesures de séparation. Or il y a des États qui ne connaissent pas de séparation formelle mais dont les formes de gouvernement sont essentiellement démocratiques, raison pour laquelle ils ne requièrent aucune légitimation ecclésiastique ou sacrée. En réalité, le cas français constitue un exemple admirable de l'existence d'une forme de laïcité sans séparation, puisque l'école laiqque s'est développée dans ce pays dans le dernier tiers du XIX siècle et la séparation a été proclamée en 1905. En d'autres termes, il existait une forme de laïcité sous 
un régime de non séparation. S'il peut exister des pays laïques sans qu'ils le soient formellement ou sans séparation entre l'État et les Églises, c'est parce que la séparation n'est une condition ni nécessaire ni suffisante pour le développement de la laïcité.

Ceci signifie également qu'il peut y avoir des pays formellement laïques, mais encore conditionnés par l'appui politique de l'une ou des Églises majoritaires du pays. À l'inverse, il existe des pays qui ne sont pas formellement laïques, mais qui, dans la pratique et pour des raisons liées à un contrôle historique de l'État sur les Églises, ne sont pas dépendants de la légitimité octroyée par des institutions religieuses.

Une autre erreur fort commune, provenant d'une interprétation non critique de la tradition française, était d'associer État laïque et République. Ce fut le cas de l'expérience hexagonale, où la Révolution puis la République se sont opposées à l'Ancien Régime représenté par la monarchie. La lutte pour la laicité, après la chute de Napoléon III en 1870, conséquence de la guerre franco-prussienne, a été menée en même temps que la bataille pour la consolidation de ce que l'on a appelé la Troisième République, raison pour laquelle les Français ont de grandes difficultés non seulement à séparer laïcité et République ${ }^{2}$, mais encore à comprendre que la laïcité puisse exister sous des formes non républicaines, bien que démocratiques, comme dans le cas de nombreuses monarchies constitutionnelles.

Par ailleurs, cette définition élargie de la laïcité permet d'observer comment, indépendamment du régime légal de certains pays, les États, c'est-à-dire l'ensemble des institutions politiques par lesquelles ils sont gouvernés, dépendent, dans une certaine mesure, de la légitimité émanant des institutions religieuses. Ainsi, les pays d'implantation protestante sont historiquement plutôt laïques, bien qu'ils aient des Églises nationales ou officielles. Dans les pays où les Églises orthodoxes sont fortement implantées, comme la Grèce ou la Russie, l'État est moins laïque, car il dépend encore pour une bonne part de la légitimité provenant de l'institution religieuse. Les pays majoritairement latins ou catholiques présentent, nous l'avons dit, une troisième variante où l'on a généralement plusieurs degrés de séparation et où la relation entre l'État, qui aspire à une autonomie de gestion, et l'Église majoritaire, qui prétend agir sur la politique publique, est une relation tendue. On a donc, dans ces cas-là, un État plus ou moins laïque selon, d'un côté, le niveau d'indépendance qu'il acquiert, et de l'autre, la légitimité qu'il tire de l'institution ecclésiastique.

De toutes les façons, postuler qu'un processus de laïcisation est amorcé lorsque l'État n'est plus légitimé par une religion ou par le sacré, mais par la volonté souveraine des citoyens, permet une nouvelle interprétation du phénomène. Cela signifie également que pour apprécier la laïcisation d'un pays, il faut

2. Le titre du "Rapport au Président de la République ", élaboré en 2004 par la Commission Stasi, s’intitule précisément Laïcité et République. 
recourir à une série d'indicateurs plus concrets, au même temps qu'aux principes fondamentaux qui la constituent ${ }^{3}$.

\section{L'héritage colonial de l'Amérique latine}

Cette définition de laïcité établit un cadre de référence qui permet non seulement de comprendre le modèle mexicain de laïcité, mais également les relations entre l'État et les Églises en Amérique latine. De même qu'en Europe occidentale, ces relations partent d'un modèle fondé sur le monopole religieux et sur le principe cujus regio ejus religio, c'est-à-dire sur l'idée des Églises officielles et nationales, privilégiées et protégées par leur roi ou leur gouvernement, ainsi que sur l'intolérance ou, dans le meilleur des cas, une tolérance limitée envers les autres religions. Rappelons dans ce sens que "la découverte de l'Amérique » a lieu en même temps que l'expulsion des juifs et des musulmans du Royaume de Grenade (1492) et que l'empire de Charles Quint (d'Espagne) coïncide avec le surgissement du protestantisme et de la Paix d'Augsbourg, le 29 septembre 1555 en Allemagne. Si l'on instaure en France le principe «d'un roi, une loi, une foi ", le développement de l'Église en Amérique ibérique s'établit sous le modèle du Patronat, cest-à-dire, du droit que le Saint-Siège concède aux rois pour fonder, administrer et du même coup contrôler l'institution ecclésiastique, afin de propager la foi sur les terres découvertes et colonisées.

Le Patronat, concédé par le Saint-Siège à travers les bulles émises par la papauté à la fin $d u X V^{e}$ siècle début $d u X V I$ siècle, octroie aux rois catholiques d'Espagne plusieurs droits sur la gestion ecclésiastique. La Bulle d'Alexandre VI, du 4 mai 1493, leur concède le domaine des Indes et le privilège exclusif de christianiser les indiens; celle du Pape, du 16 novembre 1501, garantit à la Couronne espagnole les dîmes et les premiers revenus des Églises des Indes, et la Bulle de Jules II, du 28 juillet 1508, concède au Patronat universel tous les droits sur l'Église catholique dans les Indes. Malgré quelques désaccords, au cours des siècles suivants, sur le sens de cette concession, le régalisme a assumé que ces bulles reconnaissaient l'autorité des rois d'Espagne pour exercer leur juridiction sur toutes les affaires liées au gouvernement ecclésiastique dans les Indes. Les souverains s'assumaient comme « une sorte de vicaires apostoliques, ayant autorité sur les affaires spirituelles en Amérique " (Mecham, 1934 : 14). En d'autres termes, puisque la Couronne se chargeait de tous les frais de l'expansion de la foi, le Saint-Siège admettait le droit des rois à fonder des Églises, nommer des évêques et des clercs, et à les entretenir grâce à la dîme. Il n'est pas clair - ceci fera l'objet de sévères disputes dans les siècles suivants - si cela équivalait à la reconnaissance d'un droit intrinsèque des rois ou d'une concession

3. C'est précisément à cela-mesurer l'indice de laïcité des États du monde - que je consacre actuellement mes efforts, centrés au départ sur l'Amérique latine. 
irrévocable du Saint-Siège. En tout état de cause, la Couronne espagnole assume la défense de la foi catholique et persécute, dès le début de la conquête et de la colonisation, les «idolâtries » locales et toute tentative de pénétration du judaïsme ou du protestantisme. L'Amérique ibérique devient ainsi une forteresse du catholicisme, pratiquement inexpugnable, si ce n'est la conquête de quelques petites îles des Caraibes par les Anglais et les Hollandais, qui n'affecte pas les colonies principales du continent.

Le système du Patronat renforce et accroît le régalisme de la Couronne espagnole et, par la suite, le « juridictionnalisme » des premières républiques indépendantes d'Amérique latine. La laïcité se construit dans la région dans un contexte culturel dominé par deux idées qui vont modeler le futur des nations: à partir des deux antécédents - «cujus regio ejus religio » ou «un roi, une loi, une foi $i$ - il ne viendrait à l'idée de personne, ou presque, que les nouvelles républiques puissent se passer de religion, catholique en l'occurrence, et que celle-ci ne constitue pas le fondement de la société à construire. Mais le régalisme, surtout à partir de l'arrivée des Bourbons à la Couronne (1700), présuppose une Église assujettie aux objectifs de la royauté et met en place une série de mesures politiques qui aboutissent à l'affaiblissement matériel et symbolique de l'Église en Amérique. Cette tradition est récupérée par plusieurs gouvernements des nouvelles nations indépendantes et se transforme en différentes formes juridiques ou d'intervention de l'État en matière religieuse. Loin de vouloir parvenir, au départ, à une séparation de l'Église et de l'État, leur intention visait, au contraire, la continuité du Patronat sous de nouvelles formes juridiques, et ils considéraient l'institution ecclésiastique comme une partie de l'État.

Durant les trois siècles de l'époque coloniale - de 1492 à 1821 environ, excepté pour Cuba qui reste une colonie jusqu'en 1898 - l'Église catholique ne fait pas front au pouvoir de la Couronne, puisque la relation n'est pas une relation de concurrence mais de coopération qui donne lieu, une fois le Patronat consolidé, à une relation de subordination ${ }^{4}$. L'Église est une corporation qui fait partie de l'État et ses hommes constituent un ingrédient fondamental de la classe dirigeante. Ce qui ne veut pas dire qu'il n'existe pas de différences institutionnelles, d'optiques et même d'objectifs entre les pouvoirs civil et ecclésiastique, différences particulièrement sensibles dans les moments importants de définition et de redéfinition de la structure sociopolitique, comme le sont les premières années postérieures à la Colonie, les changements de dynastie ou la fin de la vice-royauté. Mais il faut mentionner que ces désaccords éclatent au sein de l'«État » colonial et non pas entre l'État et l'Église, raison pour laquelle

4. Roberto Jaramillo mentionne un phénomène semblable: «Il est souvent répété que durant l'époque de la vice-royauté en Nouvelle Espagne, l'Église et l'État étaient étroitement unis et celui-ci obéissait aux ordres de celle-là ; dans les faits, il n'en est rien : l'Église était aux ordres de l'État, raison pour laquelle le processus de séparation entre l'Église et l'État a été très long et extrêmement complexe dans les États latins à racines catholiques. ” (1999: 13) 
l'indépendance des nouvelles nations hispano-américaines permettra au SaintSiège de récupérer son autonomie administrative et politique, perdue des siècles auparavant avec le Patronat. Cette autonomie ne va pas signifier une perte de privilèges, mais une relation plus égalitaire avec l'État, sous la logique des deux épées ou de l'Église conçue comme société parfaite. Celle-ci devra cependant faire face à deux obstacles majeurs : le désir des élites indépendantistes de renouveler le Patronat et le juridictionnalisme de ces dernières, héritées du régalisme bourbonien, qui cherche à contrôler l'appareil ecclésiastique et à l'utiliser à des fins politiques. La lutte contre l'Église ne sera pas sans incidence sur la formation d'un laïcisme combatif qui, malgré ses défauts, jettera les bases de la laïcité postérieure de l'État. J'entends par laïcisme une attitude de combat destinée, dans ce cas, à établir une laïcité. Par conséquence, laïcisme et anticléricalisme visent l'établissement de la laïcité, sans parvenir nécessairement à dépasser cet esprit de combat.

\section{Religion populaire et laïcité}

La religiosité populaire est un élément fondamental permettant d'expliquer le type de laïcité qui sera implanté dans la République mexicaine. En effet, contrairement au continent européen, en Nouvelle-Espagne, puis au Mexique, l'Église n'a jamais pu disposer du nombre de prêtres suffisant pour couvrir l'ensemble du territoire. Le type de religiosité prédominant se caractérisera donc par une doctrine assez faible et une autonomie relativement forte en ce qui concerne la gestion des fêtes et de la vie religieuse quotidienne. Malgré une grande affluence de prêtres et surtout de religieux aux XVI et XVII siècles, le clergé s'est concentré principalement dans les villes les plus importantes du centre et de l'ouest du pays ${ }^{5}$, délaissant plusieurs régions, notamment les régions côtières et montagneuses, où trouvaient refuge de nombreuses communautés indigènes ou métisses. Si celles-ci étaient formellement catholiques, elles pratiquaient une forme de religion syncrétique et surtout autonome par rapport à l'appareil clérical. Les prêtres sont encore aujourd'hui sollicités pour les sacrements, mais les communautés ne leur permettent aucune ingérence dans la gestion de la vie religieuse quotidienne du village. Il s'ensuit un catholicisme "populaire », caractérisé non seulement par une adaptation et une "refonctionnalisation » des diverses traditions religieuses, et également par l'absence d'une direction religieuse de type bureaucratique. Le vide créé donne lieu dans les campagnes, entre autres, à toute une série de prophétismes et messianismes, à peine contrôlés par une Église et un pouvoir civil qui agissent, dans la mesure du possible, ensemble. Dans la période coloniale, les procès contre des communautés accusées de paganisme et diverses déviations doctrinales sont fréquents.

5. Encore aujourd'hui, la proportion de prêtres par habitant est d'environ 1 pour 6000 , proportion bien moindre en Europe. 
Après l'Indépendance, avec les guerres civiles et l'affaiblissement de l'autorité ecclésiastique, toute l'Amérique latine connaît des messianismes politicoreligieux. Le plus célèbre est sans doute celui de la Guerre des Canudos, dans le nord-est du Brésil (1893-1897), où le prophète Antonio Conselheiro et ses milliers des fidèles contesteront simultanément les pouvoirs politique et ecclésiastique jusqu'à la destruction du mouvement. Mais il y a aussi des antécédents dans la même région, avec "le prophète "Silvestre Jose dos Santos du Pernambuco (1817) ou « le pèlerin » Joao Ferreira (1836). Au Mexique, le soulèvement du village Tomochic (1891), dans la cordillère montagneuse de Chihuahua est aussi caractéristique : la petite population, après avoir écouté Teresa Urrea, "la sainte de Cabora ", décide d'expulser le prêtre catholique local et initie une rébellion qui finira avec la destruction du village, comme cela se passera plus tard avec Canudos au Brésil ${ }^{6}$. Les exemples peuvent se multiplier. On pourrait affirmer ainsi que le développement de ce catholicisme « läqque o ou « a-clérical », voire parfois anticlérical, permettra, durant les siècles suivants, la consolidation d'un laïcisme populaire qui sera à la base des mouvements politiques séculiers du Mexique et de l'Amérique latine indépendante. On ne pourrait expliquer, sinon, l'origine des armées libérales et l'appui d'une grande partie du peuple aux libéraux contre les conservateurs et le clergé durant les guerres civiles, aussi bien au moment de la Réforme (1857-1860), que durant l'intervention française (18621867) ou la révolution (1910-1920). Ces armées seront formées tant par des artisans urbains que par des petits agriculteurs paysans, catholiques mais habitués à contester le pouvoir du prêtre et de la hiérarchie, considérée, justement, comme partie de l'établissement politique.

\section{Laïcité et pluralité : le modèle latino-américain}

Par ailleurs, la laïcité latino-américaine, là où elle a pu voir le jour, n'est pas le produit d'une pluralité religieuse qui existerait en dehors de l'Église catholique. Contrairement à l'expérience européenne, où la laïcité répond en grande partie au besoin de protéger la liberté de conscience et la pluralité confessionnelle, la laïcité ibéro-américaine est plutôt génératrice d'un espace politique et juridique qui permet la gestation d'une véritable pluralité, niée pendant trois siècles. Il ne s'agit plus de reconnaitre les cultes aux dieux préhispaniques, ni même de comprendre le mélange de rites «païens ", agricoles ou urbains, qui constituaient les pratiques catholiques de la population. Il s'agit plutôt de la possibilité d'instaurer un catholicisme sui generis, sans que l'Église, c'est-à-dire le clergé, utilise la force de l'État pour obliger les croyants à se plier au modèle romain de catholicité. Ainsi, par exemple, le facteur central qui a donné naissance à la

6. Sur la religiosité populaire en Amérique latine la bibliographie est énorme. Consulter, par exemple, José Luis Gonzáles Martínez, 1989, et 2002. 
laïcité mexicaine n'était pas la nécessité de répondre à une pluralité confessionnelle qui exigeait la tolérance, ni de réagir aux pressions exercées pour obtenir des libertés religieuses, mais une lutte pour établir les formes de la souveraineté. Tandis que l'État républicain postule la volonté populaire comme critère de définition ultime du pouvoir souverain, l'Église refuse d'accepter une souveraineté qui n'est pas engendrée par le sacré ni entérinée par le pouvoir religieux. Même ainsi, la plupart des nouvelles nations indépendantes se conçoivent dans une unité religieuse clairement inscrite dans leurs constitutions. Elles ne prétendent pas garantir la liberté de culte et encore moins promouvoir la pluralité religieuse. Le conflit, tout particulièrement au Mexique, surgira pour d'autres raisons, liées à la logique de la construction d'un État autonome, capable d'exercer sa souveraineté au-delà des intérêts particuliers ${ }^{7}$.

Ainsi, si nous entendons la laïcité comme la transition de systèmes d'autorité politique basés sur des formes de légitimité sacrée vers un État fondé sur la souveraineté populaire, nous pouvons affirmer que les premières républiques indépendantes de l'Amérique hispanique ne sont pas nées comme des nations laiques. Tout au contraire, dans tous les nouveaux pays surgis de la décomposition de l'Empire espagnol, les gouvernants ont maintenu la tradition du Patronat Royal et rédigé des constitutions qui protégeaient la religion catholique, privilégiaient l'Église romaine et ne toléraient pas la présence d'autres cultes. Le « libertador » Simón Bolivar a établi pour la Grande Colombie (Équateur, Colombie et Venezuela) la liberté des cultes durant la décennie des années 1820, mais sans éliminer le Patronat ni établir la séparation avec l'Église. Dans le cas du Brésil, la prolongation du Patronat durant l'Empire a permis la continuation du catholicisme en tant que religion d'État jusqu'à l'avènement de la République en 1889 , ce qui n'a pas empêché de grandes disputes sur la manière d'administrer le Patronat ${ }^{8}$.

\section{Patronat et juridictionnalisme}

Pour les premiers indépendantistes de l'Amérique latine, l'union entre l'Église et la patrie était une évidence. Encore plus pour les Mexicains, non seulement parce que certains "pères de la patrie » furent des prêtres, bien que libéraux, mais aussi parce que l'on considérait que la religion constituait l'ingrédient fondamental du ciment social et de l'identité de la nouvelle nation. Personne ou

7. La narration historique qui suit puise ses sources dans différentes versions, corrigées et augmentées. Voir tout particulièrement, Blancarte, 2000 et 2008.

8. Sur l'histoire général du Brésil consulter Boris Fausto et Sérgio Buarque de Holanda (eds), História Geral da civilizaçao Brasileira, 11 vols. Difusão Europea do Livro (Rio de Janiero, Bertrand Brasil, 1988-2003). Pour des événements plus récents voir Serbin (2000) et Juan Esquivel, "Laicidades relativas: Avatares de la relación Estado-Iglesia en Brasil" et "Violaciones de las libertades laicas en el Brasil del siglo XXI", in Blancarte, (coord.), 2008 : 163-192 et 193-218. 
presque ne pouvait imaginer un État séparé de l'Église catholique, ou que le catholicisme ne puisse occuper une place privilégiée dans le Mexique indépendant. Dans ce cadre, la légitimation religieuse du nouveau pouvoir politique paraissait aussi évidente que nécessaire. L'Acte d'Indépendance de Chilpancingo et les Sentiments de la Nation, rédigés par le prêtre insurgé José María Morelos y Pavón en 1813, le Décret Constitutionnel d'Apatzingan de 1814 et l'Acte Constitutif de 1823 postulaient officiellement la protection de la religion catholique et la non-tolérance des autres religions. La Constitution de 1824, la première du Mexique, postule explicitement que : « la religion mexicaine est et sera perpétuellement la religion catholique, apostolique, romaine. La nation la protège par des lois sages et justes, et interdit l'exercice de toute autre religion ". Néanmoins, le fait que les nouveaux gouvernements prétendent offrir un traitement privilégié à la religion catholique n'implique pas forcément une domination complète de l'Église. Cela signifie au contraire que l'État réclame les droits implicites du Patronat et donc un contrôle sur les activités du clergé et de l'institution ecclésiastique. En témoigne l'article 50 de cette Constitution de 1824, qui octroie au Congrès toutes les facultés pour exercer les droits du Patronat, et éventuellement, signer un Concordat avec le Saint-Siège. Aucun État laïque n'est donc en germe à cette époque, puisque les nouveaux gouvernants font de la religion l'élément central de la légitimité du nouvel État indépendant.

La question du Patronat, convertie en prétention juridictionnelle ou de contrôle sur l'Église catholique, deviendra un héritage empoisonné pour les premières républiques indépendantes de l'Amérique hispanique. Le Saint-Siège profite de la conjoncture pour réaffirmer la doctrine ultramontaine à l'égard du Patronat, en argumentant qu'il s'agit d'une concession, donc qu'elle n'est pas inhérente à la souveraineté et, en conséquence, ne peut constituer l'héritage des républiques naissantes. Celles-ci sont, au contraire, imprégnées de l'esprit régalien et supposent indispensable, pour leur identité, de maintenir la légitimation religieuse de leurs gouvernements. La tradition régalienne, qui prétendait l'assujettissement de l'Église aux objectifs des monarques, s'est renforcée à partir de l'arrivée des Bourbons en Espagne, en 1700. Mais ce fut surtout à partir de la seconde moitié du XVIII siècle, et tout particulièrement avec Charles III, que les dites " Réformes bourboniennes » lancées pour rendre l'administration coloniale espagnole plus efficace et accroître ainsi les ressources qui provenaient d'Amérique ont entraîné une modification des relations entre la Couronne et l'Église. L'époque des Augsbourg avait été dominée par la doctrine des deux épées, l'une temporelle, l'autre religieuse, qui travaillait pour un objectif commun sous la potestas ou autorité des rois d'Espagne. Cependant, l'idée d'une collaboration entre le pouvoir temporel et l'Église sous l'égide du monarque fut suivie, à l'époque des Bourbons, par la conception d'une Église instrumentalisée par la Couronne, qui devait être assujettie aux objectifs de l'Empire espagnol. Les différentes mesures politiques et administratives mises en place à partir de la seconde moitié du 
XVIII 'e siècle - l'aliénation des biens ecclésiastiques étant la plus importante d'entre elles - vont conduire à l'affaiblissement de l'Église en Amérique espagnole et constituer un premier pas dans le processus de sécularisation ${ }^{9}$ postérieure. La doctrine économique sous-jacente supposait qu'une bonne partie des terres, des biens immobiliers et des richesses dont l'Église était propriétaire (biens de mainmorte) restaient hors circuit et empêchaient les initiatives économiques. Il était donc suggéré d'aliéner les biens de l'Église, éliminer les biens de mainmorte, profiter des terrains vagues ou en jachère pour fomenter la petite et la moyenne propriété. Si tout cela ne constituait pas encore d'élément laïcisant à proprement parler, puisque les rois fondaient leur empire sur la volonté de Dieu, c'était néanmoins un antécédent important destiné à affaiblir le pouvoir temporaire de l'institution ecclésiastique. Ainsi, ce que les libéraux finirent par faire dans la seconde moitié du XIX siècle avait été, en réalité, enclenché par la Couronne espagnole, l'une des principales forteresses du catholicisme.

Par la suite, la question du Patronat allait devenir un élément de discorde non seulement entre les nouveaux gouvernements latino-américains et le SaintSiège, mais entre ceux qui défendaient une alliance entre pouvoir temporel et autorité spirituelle, caractéristique de l'ancien régime, et ceux qui commençaient à penser un schéma de séparation entre les différentes sphères d'activité. Le pouvoir de l'Église catholique au Mexique et dans de nombreux pays hispano-américains allait sérieusement être remis en question. À la base de ce questionnement, il y avait un mélange de libéralisme politique, une vision du juridique héritée du régalisme et de la pratique du Patronat, ainsi que d'énormes besoins matériels pour les nouveaux gouvernements. Ces pays étaient convaincus, depuis même l'époque coloniale, que la position dominante de l'Église empêchait l'épanouissement économique des personnes et de l'ensemble du pays, ainsi que les libertés politiques des individus. La pensée libérale, qui était diffusée à l'intérieur et à l'extérieur de la franc-maçonnerie, prêchait « la sécularisation de la source de souveraineté ». L'établissement des républiques, sous le principe de la souveraineté populaire, menait inévitablement à un affrontement autour des sources de la légitimité des institutions politiques. Le Patronat pouvait subsister, dans la mesure où il représentait le legs d'un juridictionnalisme régalien, et s'il en jetait les bases, il ne constituait pas pour autant un régime laïque, puisqu'il prétendait continuer à s'appuyer sur l'autorité ecclésiastique. Le surgissement d'une division entre conservateurs et libéraux et d'un sécularisme ou laïcisme combatif sera la conséquence d'un dilemme resté sans solution dans de nombreux pays, que celuici fasse l'objet de négociations ou de conflits armés.

Le problème au Mexique surgit lorsque le Saint-Siège refuse non seulement d'accepter l'indépendance du pays, mais encore remet en question la volonté du

9. En fait, les mots laïcité et laïcisme n'existaient pas à l'époque. On parlait par contre de la sécularisation des doctrines ou des cimetières. 
nouvel État indépendant de maintenir le Patronat. D'où l'approfondissement du conflit entre, d'une part, les premiers gouvernants mexicains qui cherchent à utiliser la figure du Patronat pour conserver un pouvoir de juridiction ou de contrôle sur les affaires de l'Église, comme la nomination des évêques, et de l'autre, la Curie romaine, qui profite des circonstances pour se libérer des contrôles établis par la Couronne à travers le Patronat.

Tout n'est pas, cependant, réductible à un conflit entre libéraux et conservateurs, comme on le dit souvent, puisque, nous l'avons vu, la majorité des créoles indépendantistes considèrent la religion catholique comme l'un des rares éléments d'identité nationale, au-delà des divisions ethniques, régionales, linguistiques, économiques, politiques ou culturelles. C'est pourquoi la protection du catholicisme et le maintien des privilèges de l'Église sont considérés comme indispensables. Mais, au fur et à mesure que pénètrent les idéaux libéraux et que le système républicain (démocratique, représentatif) gagne l'ensemble du continent, le conflit autour des formes de légitimité de l'autorité politique devient inévitable. La souveraineté populaire n'est pas vraiment compatible avec l'autorité imposée par la grâce divine. Par ailleurs, l'idéal libéral favorise l'ouverture au commerce et aux migrations européennes, ce qui conduit à une plus grande liberté de culte ou du moins à la tolérance à l'égard des non-catholiques. Les besoins financiers des gouvernements et de la population mènent également à des mesures destinées à affaiblir le pouvoir économique et politique de l'Église, comme la nationalisation des biens ecclésiastiques, l'élimination de la dîme ou l'abolition du for ecclésiastique. Finalement, après des années de débats et de frustration, la nouvelle élite gouvernante en vient, peu à peu, à l'idée qu'une séparation entre les affaires de l’Église et celles de l'État était la meilleure solution.

La dynamique régalienne et juridictionnelle, léguée par le Patronat, et le surgissement du libéralisme politique menèrent à des conflits ouverts entre les libéraux et les conservateurs, ces derniers étant soutenus par la hiérarchie catholique. Après la victoire des libéraux, les mesures tendront plus à soumettre l'Église qu'à séculariser la société. Il s'agira davantage d'utiliser la religion comme outil que de laïciser les institutions politiques. On est donc face à un conflit politique (et parfois militaire) qui viendra de l'impossibilité de rallier l'Église aux côtés de l'État, même si, dans certains cas, il pourra éventuellement déboucher sur des mesures politiques de séparation ou de laïcisation. Là encore, il s'agira de laïcisme et non de laïcité.

Le premier libéralisme mexicain de la première moitié du XIXe siècle connaît alors quelques affrontements avec l'Église, durant des expériences généralement courtes de gouvernement, interrompues par des réactions conservatrices. En 1833, le vice-président Valentín Gomez Farías, en l'absence du Président Antonio López de Santa Ana, de fait rarement présent, essaie de mettre en marche le programme libéral : interdiction pour l'Église d'acquérir des biens immobiliers, élimination de la dîme (c'est-à-dire de l'obligation civile de la payer), suppression 
des institutions monastiques (et de la contrainte civile pour l'accomplissement des vœux) et en général de "toutes les lois qui attribuent au clergé la connaissance des négoces civils, comme le contrat de mariage, etc. ». On rappelle aux autorités ecclésiastiques l'observation des dispositions qui interdisent au clergé séculier et régulier d'aborder ou de se prononcer officiellement sur les affaires politiques. On cherche, de même, à rompre le monopole éducatif de l'Église, depuis l'école primaire jusqu'à l'université, monopole supprimé et remplacé par une Direction Générale de l'Instruction Publique, responsable de l'enseignement dans le pays. Cependant, la plupart de ces mesures, à l'exception des mesures économiques, ne parviennent pas à être mises en pratique et sont contrées par les gouvernements conservateurs successifs. Même situation en 1847, lorsque Gomez Farias, de nouveau Président et en pleine guerre contre les États-Unis, essaye sans succès d'exproprier les biens ecclésiastiques. Il faudra attendre la Révolution d'Ayutla, en 1855, pour que le programme libéral commence à avancer, avec de grandes difficultés, au milieu de révoltes et de guerres, comme celles de la Réforme (1857-1860) et de l'intervention française (1862-1867).

\section{Clergé et libéralisme}

Les débats ne concernent pas seulement la société politique de l'époque, mais également les membres du clergé en Europe et en Amérique hispanique. Cette lutte interne à l'Église va définir ses rapports avec le libéralisme et le monde moderne. Durant la première moitié du XIXe siècle, nombreux sont les clercs, sur les deux continents, qui cherchent à concilier libéralisme et catholicisme. Mais le résultat final leur est adverse. N'étant pas disposés à accepter les conséquences que cette nouvelle situation entraînerait pour l'Église, les partisans de la nonconciliation l'emportent. Les États Pontificaux sont pris dans le tourbillon des révolutions bourgeoises de la moitié du siècle et conduisent le Saint-Siège à s'enfermer dans une intransigeance croissante et à condamner le libéralisme et ses conséquences en matière de religion. Mais durant les premières décennies des pays indépendants, les membres du clergé sont des acteurs de premier plan dans les différents débats, aussi bien du côté du nouveau régime que de l'ancien. Plusieurs d'entre eux défendent un républicanisme catholique et les droits de l'Église dans le système libéral. Des travaux récents, comme ceux de Brian Connaughton (2005), montrent que, même jusque dans les années 1850, de nombreux clercs s'efforcèrent de démontrer que le catholicisme et le progrès - Les Lumières ou la science - n'étaient pas incompatibles.

Au Mexique, le litige est finalement résolu de manière drastique. Les libéraux mexicains se rendent compte que la question du Patronat est sans issue et que la seule solution est peut-être l'adoption d'un régime de séparation où l'Église (à cette époque il n'y en avait qu'une) s'occuperait de ses affaires et l'État des siennes. La Constitution libérale de 1857 déclare de fait la séparation, en omettant tout simplement les traitements privilégiés de l'Église ou l'intolérance envers 
les autres religions. La Guerre de trois ans (ou Guerre de Réforme) entre les libéraux et les conservateurs radicalise les positions à un point tel que les Lois de Réforme sont promulguées entre 1859 et 1860 . Celles-ci décrètent la nationalisation des biens ecclésiastiques, la séparation entre l'Église et l'État, la création d'un Registre, le mariage civil et la sécularisation des cimetières. En d'autres termes, l'ensemble des mesures imposées par les Lois de Réforme sépare, comme on le disait à l'époque, "les négoces ecclésiastiques " et les "négoces de l'État ". Mais elles permettent surtout la constitution des institutions essentielles à tout État laïque, c'est-à-dire, indépendant des institutions ecclésiastiques. La séparation permet le début de la formation d'un État, dont les institutions n'allaient plus dépendre de la légitimité religieuse.

\section{Benito Juárez et le modèle mexicain de laïcité}

Au cours de ces années marquées par des affrontements et des décisions politiques difficiles, bien que nécessaires pour les libéraux, la figure de l'indigène zapotèque, Benito Juarez, allait s'imposer au sein d'une brillante génération de libéraux. Ce grand homme d'Oaxaca est indéniablement le fondateur de l'État laïque mexicain. La «Loi Juarez » de 1855, qui commence par éliminer timidement le for ecclésiastique dans l'administration civile, même s'il subsistait encore en matière de criminalité, devait être le début d'une série de mesures qui aboutiront à l'établissement d'institutions civiles essentielles pour la construction de l'État moderne. Les actions entreprises par Benito Juarez, tout particulièrement les Lois de Réforme promulguées en pleine guerre contre les conservateurs (18581860), prennent place dans un contexte général caractérisé par un mélange de juridiction régalienne et de libéralisme qui finit par affaiblir la vieille structure de pouvoir liant étroitement les monarchies au Saint-Siège. Dans les deux cas, les pouvoirs séculiers ou civils sont renforcés au détriment de l'Église, mais plutôt que la constitution d'un espace d'autonomie laïque, ceci implique l'assujettissement du clergé aux dictats du pouvoir civil, qu'il soit monarchique ou républicain. Juarez allait fonder un autre modèle de laïcité distinct, dans son essence, du simple prolongement du Patronat ou de l'annihilation de l'Église. Il allait vers la constitution d'une république, où il n'y aurait « qu'une seule autorité : l'autorité civile définie par la volonté nationale, sans religion d’État, moyennant la disparition des pouvoirs militaires et ecclésiastiques ${ }^{10}$.

Le modèle de laïcité établi par les libéraux mexicains reposera principalement sur ce que j'ai identifié comme son pilier, c'est-à-dire, sur la légitimité du pouvoir

10. Benito Juárez, "Apuntes para mis hijos", en Documentos, discursos y correspondencia. Selección y notas Jorge L. Tamayo. Héctor Cuauhtémoc Hernández Silva, coordinador de edición digital. México, DF, Secretaría de Cultura del Gobierno del DF y Universidad Autónoma Azcapozalco, 2006, tomo I, capítulo 1, pp. 14-15. 
politique. Contrairement aux autres formes de rapports entre l'État et les religions du monde, qui exprimaient encore à cette époque le besoin social du religieux et qui jugeaient donc important de maintenir un lien entre le religieux et le politique, le modèle mexicain de laïcité cherchera simplement la constitution d'un pouvoir civil autonome de la religion. Mais, de par ses liens historiques avec le Patronat et le régalisme, il cherchera à imposer son autorité tout en abandonnant formellement le désir de diriger en s'appuyant sur le sacré ou sur les institutions religieuses. Au moment où Juarez et les libéraux décrètent les Lois de Réforme (1859-1860) aux fondements de l'État laïque, il n'existe pratiquement pas de modèles de laïcité sur lesquels s'appuyer. La Révolution française, avec ses mesures laïcistes et anticléricales, constitue certainement un exemple à suivre respecté par beaucoup d'entre eux. Le bonnet phrygien apparaît fréquemment au sein des symboles républicains des pays latinoaméricains, et de nombreux écrits des Lumières et du libéralisme français sont lus par les élites hispano-américaines. Le président Benito Juarez, au moment de sa mort, en 1872, aura sur sa table de chevet le Cours d'histoire des législations comparées de Jean-Louis-Eugène Lerminier (1803-1857), grand professeur libertaire du Collège de France, proche à une époque de l'école saint-simonienne et cité par Pierre-Joseph Proudhon, fondateur de l'anarchisme. Melchor Ocampo, une autre des grandes figures du libéralisme mexicain, est dépeint par ses hagiographes comme un "disciple de Rousseau et élève de Proudhon » ${ }^{11}$. Mais, si la Révolution française constitue un modèle à suivre pour les libéraux mexicains, la France à proprement parler, régie par un modèle de cultes reconnus depuis Bonaparte et mis en pratique de manière très fonctionnelle par Napoléon III, n'en est pas un. En effet, pour Juarez et les libéraux de son époque, le modèle français ne peut être mis en pratique au Mexique, pays où il s'agit avant tout de combattre le pouvoir de l'Église monopoliste et non de garantir une liberté de cultes qui, pour la plupart, n'y existent pas. Le modèle des cultes reconnus ressemble trop à celui que l'on a voulu établir avec le Patronat, avec des Églises et des religions au service de l'ordre social et politique, à la différence qu'au Mexique ou en Amérique latine, il n'y a qu'une Église et que celle-ci domine.

Les autres modèles sont également lointains. Le modèle américain peut paraître relativement attirant dans la mesure où le premier exemple de séparation entre l'État et les Églises avait justement vu le jour dans les colonies anglaises qui venaient d'obtenir leur indépendance et où le libéralisme présentait un franc succès. Mais le modèle ne peut être complètement adopté, car les Américains s'appuient sur l'existence d'une pluralité religieuse pour engendrer une religion civile, liée à un destin manifeste. Alexis de Tocqueville allait l'exprimer clairement : la liberté religieuse était, en Amérique, le fondement de la république démocratique. C'était précisément ce que le nouveau régime mexicain voulait

11. Justo Sierra, Juárez, su obra y su tiempo, (México, J. Ballesca, 1905-1906), p. 93. 
éviter : l’État ne devait pas prendre appui sur une légitimité émanant du religieux, mais dépendant, au Mexique, de la bonne volonté d'une seule hiérarchie ecclésiastique. C'est pourquoi la laïcisation devait être plus radicale et plus complète. Finalement, le régime de séparation établi fut l'un des plus perfectionnés, tout du moins formellement, puisque tout en mettant fin à la pratique d'un État qui était le bras séculier de l'Église, il ne prétendit jamais utiliser la religion à des fins politiques et sociales, comme le firent d'autres gouvernements en Amérique latine et en Europe. Il s'agissait avant tout, comme on le disait à l'époque, d'une parfaite séparation entre les affaires ecclésiastiques et les affaires civiles.

\section{Le triomphe (temporaire) du libéralisme en Amérique latine}

Le libéralisme allait finalement l'emporter dans tout le monde occidental durant la seconde moitié du XIX ${ }^{e}$ siècle et au début du XXe siècle. En matière religieuse, cela impliquait la normalisation de la liberté de conscience, de croyance et de culte et l'autonomisation du politique à l'égard du religieux, même si les modalités juridiques qui les consacraient étaient plurielles. En Amérique latine en général, et tout particulièrement au Mexique, ces processus ont donné lieu à un début de pluralité religieuse. Mais le processus de laïcisation des institutions publiques sera plus complexe, dans la mesure où de nombreux États, comme l'Argentine, le Brésil et le Mexique s'appuieront sur l'autorité (et donc la légitimité) de l'Église catholique. Dans la plupart des cas, les régimes oligarchiques fondés sur des modèles économiques d'exportation agricole, idéologiquement libéraux ou libéraux-conservateurs chercheront, pour des raisons plus économiques que politiques, à empiéter sur les propriétés ecclésiastiques, afin de mettre sur le marché des terres et des biens, ce qui reviendra également à contrôler le pouvoir de l'Église. Dans bien des cas, comme en Colombie, au Chili ou dans plusieurs pays d'Amérique Centrale, ces mesures seront précédées par des affrontements armés ou des guerres civiles entre conservateurs et libéraux. Mais, plus la fin du siècle approchera, plus il deviendra évident que les oligarchies des deux camps chercheront à imposer un ordre pour le progrès, fondé sur des régimes autoritaires, éclairés, influencés par le positivisme et l'idéal scientifique de la modernité. Le Brésil républicain, à partir de 1889 , avec son drapeau qui préconise "l'ordre et le progrès ", est un symbole notoire de cette idéologie. L’Église apparaitra dans ce contexte comme une institution nécessaire pour la conservation de l'ordre moral et un obstacle pour le progrès social.

Les idéaux de liberté seront liés à la nécessité impérieuse de promouvoir la distribution de la richesse (éliminant ou limitant les biens de mainmorte), à la conviction que le pouvoir politique ne peut se partager (on est en présence de régimes non démocratiques) et à un anticléricalisme militant. Des mesures comme l'expulsion continue des jésuites (considérés comme les principaux défenseurs du Saint-Siège), la fermeture de couvents ou tout simplement l'interdiction 
d'entrer dans un monastère, deviendront courantes. Le libéralisme positiviste, introduit assez tôt au Mexique, considérait, par exemple, qu'aucun être humain n'avait le droit de renoncer à sa liberté, raison pour laquelle la loi interdisait les ordres religieux conventuels. La confiscation de terres et biens immobiliers appartenant à l'Église était également justifiée pour des raisons économiques. Quand l'Église opposera des résistances, l'anticléricalisme se montrera plus d'une fois excessif. Il n'empêche que la hiérarchie catholique, à quelques exceptions près, dont le Mexique, l'Uruguay et plus tard Cuba, ne quitte pas la sphère du pouvoir dans les pays latino-américains. Le peuple perçu comme presque entièrement catholique et la logique corporatiste prévalant, les évêques, aux côtés des militaires et des gouvernants seront perçus comme les garants de l'ordre social.

Les mesures combatives laïcistes ne parviennent à construire qu'une pseudo laïcité étatique, puisque la transition des formes d'autorité sacrée à une souveraineté populaire n'est pas complètement réalisée. Les régimes ne cessent de dépendre de l'appui de l'Église catholique, pas plus qu'il ne se crée de gouvernements véritablement démocratiques, fondés sur la volonté populaire. L'autoritarisme et la manipulation politique, le caudillisme ou la répression sont une constante en Amérique latine durant l'époque de la domination des régimes oligarchiques. Si le «laïcisme " était prédominant, la «laïcité » restait encore à construire.

Au Mexique, cependant, la défaite du conservatisme et, donc, de l'Église catholique est beaucoup plus complète et plus longue. Contrairement à d'autres pays latino-américains, la défaite des conservateurs au Mexique est totale, surtout après l'échec de l'intervention française et le piètre empire de Maximilien (1862-1867). Après la mort de Juárez, le Président Lerdo de Tejada incorpore, en 1873, les Lois de Réforme à la constitution. À l'époque porfirienne (18761910), bien que le régime maintienne son caractère libéral et laïque, survient un retour relatif aux formes de légitimation religieuse, quand Porfirio Diaz accepte une réconciliation avec la hiérarchie catholique. Mais de toutes les façons, et même si la question n'a pas encore été étudiée à fond, le fait que le régime porfirien soit resté, aussi bien dans la forme que sur le fond, plutôt libéral a constitué un facteur important de distanciation entre l'Église et le régime (on peut évoquer à ce propos le tracé de l'" Avenue de la Réforme ", une sorte de Champs Elysées en hommage aux libéraux mexicains) ${ }^{12}$. Mentionnons également

12. Le Gouvernement de Porfirio Díaz (1876-1910), par exemple, n’a jamais établi de relations diplomatiques formelles avec le Saint-Siège. En fait, comme Jean Baubérot l'a montré dans sa préface à l'édition en espagnol de son Histoire de la laïcité française, les républicains français de la fin du XIXe siècle étaient impressionnés "par les résultats merveilleux de cette législation" (Historia de la laicidad francesa (Zinacantepec, México, El Colegio Mexiquense, 2005, p. 17)). Plus tard, le Rapport Briand, 1905, présente le Mexique comme le meilleur exemple de laïcité dans le monde: "Le Mexique possède ainsi la législation laïque la plus 
que le libéralisme triomphant a très certainement contribué à un renforcement de l'État laïque, moyennant la diffusion de valeurs et de libertés qui allaient audelà des normes et des doctrines ecclésiastiques, donnant lieu très souvent à une sacralisation de l'État libéral. Ainsi, les saints sont remplacés par les héros indépendantistes et libéraux, et les autels religieux par ceux de la patrie. Si la substitution de rituels religieux par des cérémonies civiques fait apparaitre la volonté de changement sur le plan des symboles, elle est également l'indice d'une difficulté à générer des institutions véritablement laïques, c'est-à-dire, désacralisées.

\section{Révolution et laïcité}

La révolution mexicaine de 1910-1920 devait accentuer la spécificité de l'expérience mexicaine vis-à-vis des autres expériences latino-américaines. En effet, la révolution, tout particulièrement celle qui provenait du nord du pays et qui a finalement triomphé, est caractérisée par un anticléricalisme radical. Indépendamment des raisons invoquées par le radicalisme révolutionnaire à l'égard de l'Église, comme le soutien de la hiérarchie et du Parti catholique national au chef militaire contre-révolutionnaire Victoriano Huerta, les mesures imposées par la Constitution de 1917 cherchent à annihiler le pouvoir religieux dans la nouvelle société que l'on prétendait construire. La non-reconnaissance juridique des Églises, l'interdiction pour celles-ci de posséder des biens, les limites imposées au culte à l'extérieur des temples, l'éducation laïque à l'école primaire, l'impossibilité de constituer des partis politiques avec des références religieuses et les interdictions pour les ministres du culte de participer aux activités politiques ou de se prononcer contre le gouvernement ou ses lois, sont des mesures anticléricales (non anti-religieuses) qui allaient conduire en dernière instance à l'élimination de l'Église catholique du champ sociopolitique. Dans ce sens, elles ont contribué, peut-être même à leur insu, à la construction et au renforcement de l'État laïque mexicain, c'est-à-dire à un système politique qui a ignoré, durant tout le $\mathrm{XX}^{\mathrm{e}}$ siècle, les formes de légitimation provenant des institutions religieuses. Rien d'étonnant, donc, à ce que la hiérarchie ecclésiastique ait réagi avec une fermeté et une intransigeance peu communes aux mesures anticléricales, et que le radicalisme des révolutionnaires et des catholiques intégristes ait abouti à des affrontements politiques et armés comme la Christiade (1926-1929). Ce qui était en jeu, était un système sociopolitique et les fondements de l'autorité dans le nouvel État.

complète et la plus harmonique qui ait jamais été mise en vigueur jusqu’à ce jour ». Aristide Briand, Rapport fait le 4 mars 1905 au nom de la Commission relative à la séparation des Églises et de l'État et de la dénonciation du Concordat chargé d'examiner le projet des lois et les diverses propositions des lois concernant la séparation des Églises et de l'État. En http:/l www.laicite-laligue.org-1905, la séparation. 
Finalement, après un accord implicite (un modus vivendi établi en 1938) reposant sur des valeurs communes (nationalisme, recherche de justice sociale, opposition au socialisme radical), le Mexique connait l'émergence d'un État qui, s'il continue à contrôler l'espace public, surtout sur le terrain sociopolitique (syndicats, partis, organisations de masse), permet à l'Église une plus grande liberté dans le domaine éducatif, sous des formes diverses de tolérance.

Indépendamment des moments sporadiques au cours desquels l'État mexicain a recours au soutien politique de l'Église, il poursuit durant les sept premières décennies du XXe siècle son processus de laïcisation sans s'appuyer sur les institutions ecclésiastiques. Mais, encore une fois, il s'agit plus de laïcisme que d'une véritable laïcité. C'est pourquoi, au fur et à mesure que s'affaiblit l'État de la Révolution mexicaine et qu'il perd les bases de légitimité qui lui ont donné naissance, les gouvernements du parti révolutionnaire institutionnel (PRI) doivent recourir à d'autres formes de légitimité pour rester au pouvoir. Cette situation, sur laquelle viennent se greffer d'autres phénomènes internes à l'Église catholique, conduit, entre autres, à une présence publique croissante de la hiérarchie et des ministres de culte catholiques et à une pression sur le système politique pour une libéralisation, voire l'élimination, des lois anticléricales.

Les amendements de 1992 aux articles anticléricaux de la Constitution, importants sans aucun doute dans le cadre d'une reconnaissance croissante des libertés et des droits de l'homme, permettent le retour des Églises (au pluriel, cette fois) dans l'espace public. Bien que le principe de séparation entre l'État et les Églises et que l'esprit libéral de la Constitution soient maintenus, les institutions ecclésiastiques, et tout particulièrement celles de l'Église catholique, ont considéré qu'il était opportun d'agir sur la définition des lois et des politiques publiques de la nation. Plusieurs amendements constitutionnels ont été approuvés en 1992 et les aspects les plus radicaux, comme l'impossibilité pour les Églises d'avoir une personnalité juridique, la possession de biens matériels ou d'institutions éducatives, ont été éliminés. Mais d'autres principes anticléricaux sont conservés, ainsi que l'esprit libéral de la Constitution. Même si elle n'est pas satisfaisante pour tous, et en particulier pour les membres de la hiérarchie catholique, le compromis que ces mesures représentent semble pouvoir durer. L'arrivée au pouvoir d'un gouvernement idéologiquement conservateur en 2000 a ébranlé les structures laïques de l'État mexicain (c'est-à-dire les sources de légitimité de l'ensemble des institutions politiques qui le composent), phénomène particulièrement sensible dans la dernière décennie du XX⿳⺈冂大 siècle.

\section{Le cadre juridique actuel de la laïcité mexicaine}

Bien que la laïcité ne soit pas inscrite dans la Constitution mexicaine, aussi bien l'État laïque que l'école laïque sont clairement perçus et défendus par la 
population. En effet, seul un passage de l'article 3, concernant l'éducation, spécifie : "étant donnée la liberté de croyances garantie par l'article 24, l'éducation sera laïque et restera donc étrangère à toute doctrine religieuse. » Par ailleurs, dans l'article 130, concernant les groupements religieux et leurs ministres de culte, il est signalé que "le principe historique de séparation des Églises et de l'État» oriente les normes contenues dans ce dernier.

La laicité de l'État mexicain est curieusement stipulée dans une loi secondaire (par rapport à la Constitution), la Loi des associations religieuses et du culte public. Cette loi offre une sorte de définition de contenu lorsqu'elle affirme: «L'État mexicain est laïque. Il n'exercera son autorité sur toute manifestation religieuse, individuelle ou collective, qu'en ce qui concerne le respect des lois, le maintien de l'ordre et de la morale publiques et les tutelles des droits des tiers ». La loi stipule également que l'État «ne pourra établir de préférence ou de privilège d'aucune sorte en faveur d'une religion particulière » ni ne se prononcera "pour ou contre une église ou un groupement religieux d'aucune sorte".

En résumé, la laïcité actuelle mexicaine est définie par la recherche d'une séparation des champs, nettement marquée dans le domaine éducatif, un "non interventionnisme » sur le marché religieux (on ne peut parler ici de "neutralité » parce que l'État laiqque défend des valeurs comme la démocratie, la pluralité religieuse, la tolérance, etc., il n'est donc pas neutre, mais impartial) et un « juridictionnalisme » (héritage du Patronat) sur les effets sociaux des manifestations religieuses. Si cette laïcité n'est pas inscrite dans la Constitution, elle repose sur l'idée plus concrète d'" un principe historique de séparation " entre les Églises et l'État, renvoyant à une longue tradition d'un siècle et demi de régimes libéraux ou socio-radicaux.

Plusieurs propositions ont été faites, dans les dernières années, pour introduire formellement dans la Constitution, tout particulièrement dans son article 40 , le caractère laïque de l'État, et il a été procédé à des modifications importantes de l'article 130, relatif à l'État mexicain, aux Églises et aux groupements religieux.

\section{Conclusion}

La laïcité mexicaine partage avec d'autres laïcités dans le monde la recherche d'un État autonome de la religion, dans le but de garantir la liberté de conscience, ainsi que l'égalité de tous, croyants et non-croyants, face à la loi. Le passage du sacré à des formes démocratiques de légitimation du pouvoir politique est au centre de cette quête. Dans le cas du Mexique, le processus a commencé, mutatis mutandis, à l'époque de la monarchie des Bourbons, moyennant l'assujettissement du pouvoir ecclésiastique au pouvoir royal. L'héritage du Patronat s'est maintenu, durant la première étape indépendante, tant au Mexique que dans l'ensemble des républiques latino-américaines. Dans certains cas, comme en 
Amérique Centrale, au Chili ou en Argentine, les libéraux ont pu soumettre, durant quelques temps, l'Église aux dictats du nouvel État, dans d'autres, comme la Colombie ou l'Équateur, les conservateurs ont réussi à contrecarrer ces mesures et à pérenniser le modèle de l'ancien régime, dans lequel l'Église est le pilier du pouvoir politique. Dans les deux cas, et dans presque tous les pays de l'Amérique latine (sauf, peut-être au Mexique et en Uruguay), on se trouve plutôt en présence de laïcisme que de laïcité : l'État prétend continuer à utiliser le religieux pour légitimer le pouvoir politique. Le Mexique de Benito Juarez jette les bases d'un État véritablement autonome de toute forme de légitimation religieuse, et constitue en cela une exception latino-américaine. Le libéralisme des réformateurs mexicains, tout du moins formellement, refuse d'utiliser le religieux pour asseoir le pouvoir politique. Il cherche simplement la suprématie du pouvoir civil et, pour ce faire, établit ce qu'il appellera « une séparation parfaite entre les affaires civiles et celles de l'Église. » Dans le cas de l'Uruguay, la relative faiblesse historique de l'Église catholique permet l'établissement d'un État radicalement laïque.

Ce qui différencie également le Mexique du reste de l'Amérique latine, c'est que les libéraux y ont infligé une défaite radicale aux conservateurs, alliés traditionnels de l'Église catholique. Dans les autres pays, en effet, les victoires des uns et les défaites des autres ont été plus mitigées, ouvrant la porte à de fragiles accords, voire même à des reculs, surtout après la crise des oligarques agroexportateurs locaux dans les années trente. Le nationalisme populiste, marqué par le catholicisme et une nouvelle alliance entre l'État et l'Église catholique au Brésil avec Getulio Vargas et en Argentine avec les diverses dictatures militaires, montre bien ce processus ${ }^{13}$. Au Mexique, le libéralisme du XIX ${ }^{\mathrm{e}}$ siècle a été glorifié, même si dans la pratique le laïcisme a toujours eu tendance à utiliser le religieux comme élément de son pouvoir politique et social. Il n'a donc pas été nécessaire de passer des accords ou de négocier avec les secteurs conservateurs ni avec l'Église.

La révolution mexicaine, traversée par les courants positivistes, anticléricaux et sociaux-radicaux, devait confirmer la tendance. Pour des raisons diverses, les révolutionnaires ont vu dans l'Église catholique l'un de ses pires ennemis et ont décrété sa disparition comme institution politique et sociale, dans la Constitution de 1917 qui reprenait l'esprit libéral de 1857 en y ajoutant plusieurs articles anticléricaux. Le programme révolutionnaire a signifié également l'établissement d'une école publique laïque, étrangère à toute croyance religieuse, ce qui allait devenir fondamental pour la génération de valeurs nationales séculières. Mais même ainsi, il s'agissait plus d'une politique laïciste, destinée à contrôler le pouvoir clérical, que d'une réaffirmation d'une laïcité ayant pour objectif la garantie

13. Voir Stefano et Zanatta, 2000, pp. 350 et 405 ; Verónica Giménez Beliveau, "Jerarquías eclesiásticas, nación y espacio público en Argentina" ; Fortunato Mallimaci, "Nacionalismo católico y cultura laica en Argentina", in Roberto Blancarte, 2008 : 219-237, 239-262. 
de la liberté de conscience des individus, le respect des croyances diverses et la non-discrimination. La hiérarchie catholique comprend parfaitement qu'il s'agit d'une lutte pour le pouvoir, raison pour laquelle elle s'oppose à la Constitution de 1917 dès sa promulgation. De son point de vue, l'État mexicain n'avait pas le droit de limiter des droits que l'Église considérait comme inaliénables.

Mais si le laïcisme, qui est à la base des premiers pas de la laïcité mexicaine, est le produit d'un libéralisme et d'un radicalisme révolutionnaires toujours triomphants (au moins de 1855 à 2000), il est également dû au type particulier de religiosité engendrée par la conquête et l'évangélisation espagnole en Amérique, qui ont finalement donné lieu à un catholicisme superficiel, syncrétique et surtout peu cléricalisé, sauf dans certaines régions. Il serait difficile, sinon, d'expliquer l'appui populaire aux armées libérales, ainsi qu'à de nombreuses mesures anticléricales prises par les gouvernements locaux et fédéraux, durant les guerres du milieu du XIX ${ }^{\mathrm{e}}$ siècle et la révolution au XXe siècle. L'anticléricalisme militant du laïcisme mexicain s'explique en partie par une Église paradoxalement absente et éloignée de ses fidèles et par la permanence de la victoire libérale et révolutionnaire.

En fait, le plein développement de la laïcité mexicaine et latino-américaine ne date que de la seconde moitié du XXe siècle. De mon point de vue, trois facteurs ont permis la réactivation de la laïcité : 1- la gestation d'une pluralité religieuse effective et significative; 2 - une plus grande conscience du besoin de protéger les droits de l'homme et, donc, les droits des minorités au sein d'une diversité de plus en plus reconnue; 3- la démocratisation progressive mais réelle de l'ensemble des sociétés latino-américaines.

En effet, tandis qu'en Europe l'État laïque surgit d'une nécessité de tolérance après les guerres de religion, les Églises et les différentes confessions religieuses n'ont pu s'implanter en Amérique latine qu'une fois les lois de liberté de cultes décrétées, plusieurs décennies après l'Indépendance. Le monopole de l'Église catholique n'a été réellement mis à l'épreuve que dans la seconde moitié du $\mathrm{XX}^{\mathrm{e}}$ siècle. Si le pourcentage de non-catholiques est encore minimum en 1950 (il oscille entre 1 et $2 \%$ au Mexique), il passe au début du XXe siècle à environ $15 \%$ dans plusieurs pays latino-américains dont le Mexique. Cette nouvelle pluralité doit forcément être prise en compte dans la définition des lois et des politiques publiques. Le besoin de créer un État indépendant des normes religieuses particulières devient impératif pour garantir, entre autres, l'égalité des Églises face à la loi et la non-discrimination religieuse.

L'ébranlement des régimes autoritaires, comme celui du PRI et de l'État révolutionnaire au Mexique, s'est accompagné de l'intériorisation des droits de l'homme dans l'ensemble les sociétés latino-américaines, et donc d'une défense croissante de la liberté de conscience et des libertés civiques qui lui sont liées. 
Ainsi, par exemple, le droit des femmes à décider de leur corps et celui des couples à avoir le nombre d'enfants qui leur convient ou à différer leur naissance ou encore celui des adolescents à avoir une éducation sexuelle sont relativement garantis dans la mesure où la notion de liberté de conscience s'étend et que des lois sont créées ou interprétées de manière plus libérale pour protéger cette liberté ou les actions qui en découlent. C'est le cas de l'action de plusieurs Cours Suprêmes de Justice (Mexique, Colombie) dans la dépénalisation de l'avortement. La reconnaissance de l'immense diversité de la société se traduit aussi, de plus en plus, en garanties et en droits pour les minorités ethniques, religieuses, sexuelles, etc., indépendamment de la volonté des majorités, ce que montre, par exemple, les droits reconnus aux populations indigènes.

L'arrivée des régimes véritablement démocratiques en Amérique latine, surtout depuis les années quatre-vingts, a signifié une accentuation du passage des formes de légitimité fondée sur le sacré à des formes d'autorité politique basées sur la souveraineté populaire, aspect principal de la laïcité. Au fur et à mesure que l'exigence de transparence et d'imputabilité augmente, l'autorité politique est de plus en plus sujette à la volonté des citoyens. Il s'ensuit des États plus laiques - qu'ils s'identifient ou non comme tels -, qui répondent plus aux nécessités plurielles et aux exigences des nouvelles cosmovisions des divers secteurs sociaux qu'à une doctrine religieuse particulière. Malgré les traditions et les pressions politico-ecclésiastiques, l'État mexicain, comme celui d'autres pays latino-américains, est obligé de se läiciser. On peut donc affirmer que, malgré une histoire accidentée dans une région qui aura connu le régalisme, le juridictionnalisme, l'anticléricalisme et d'autres formes de laïcisme, l'Amérique latine semble finalement, au XXI siècle, découvrir la laïcité. Au Mexique, le laïcisme combatif, allié le plus souvent d'un État autoritaire, est sévèrement remis en question. Et, dans la mesure où celui-ci a jeté les bases d'une véritable transition des formes de légitimation politique, il cède le pas à une laïcité plus réelle et compréhensive.

Roberto BLANCARTE El Colegio de México blancart@colmex.mx 


\section{Bibliographie}

BLANCARTE Roberto, 2000, "Retos y perspectivas de la laicidad mexicana", in Blancarte R., Laicidad y valores en un Estado democrático, México, DF, El Colegio de MéxicoSecretaría de Gobernación, pp. 117-139.

-, 2007, "Mexico: A Mirror for the Sociology of Religion", in Beckford J.A., Demerath III

N.J., (eds.), The Sage Handbook of the Sociology of Religion, London, Sage, pp. 710-727.

-, 2008a, "Laicidad y laicismo en América latina", Estudios Sociológicos, XXVI-76, pp. 139-164.

-, 2008b, Para entender el Estado laico, México, DF, Nostra Ediciones.

Blancarte Roberto, (ed.), 2008, Los retos de la laicidad y la secularización en el mundo contemporáneo, México, DF, El Colegio de México.

Connaughton Brian, 2005, "The Enemy Within: Catholics and Liberalism in Independent Mexico 1821-1860", in Rodríguez J.E.O., The Divine Charter, Oxford, Rowman \& Littlefield Publishers, pp. 183-204.

Di Stefano Roberto, Zanatta Loris, 2000, Historia de la Iglesia Argentina: Desde la Conquista hasta fines del siglo XX, Buenos Aires, Grijalbo-Mondadori.

HaArscher Guy, 1998, La laïcité, Paris, PUF, coll. "Que sais-je?».

JARAMILLo Roberto, 1999, "El Regio Vicariato y las reformas del siglo XVIII", in Galeana P., (ed.), Relaciones Estado-Iglesia: Encuentros y desencuentros, México, DF, Archivo General de la Nación.

Gonzáles Martínez José Luis, 1989, El Huanca y la Cruz. Creatividad y autonomía en la religión popular. Lima, Tarea.

-, 2002, Fuerza y Sentido: El catolicismo popular en los comienzos del siglo XXI, México, Ediciones Dabar.

Mecham John Lloyd, 1934, Church and State in Latin America: A History of PoliticoEcclesiastical Relations, Chapel Hill, The University of North Carolina Press.

SERBIN Kenneth P., 2000, Secret Dialogues: Church-State Relations, Torture, and Social Justice in Authoritarian Brazil, Pittsburg, University of Pittsburgh Press. 


\section{Résumé}

La laïcité mexicaine s'est construite en résistant à une Église catholique omniprésente dans le tissu politique et culturel. Son influence s'affaiblira cependant progressivement dans le domaine social. Quelques particularités communes à l'ensemble de la région latino-américaine, comme le nombre insuffisant de prêtres et la prédominance d'une religiosité populaire, ajoutées à des particularités nationales, comme la victoire des forces libérales jamais mise en question depuis la moitié du XIXe siècle, contribuent à la formation d'un lä̈cisme militant lié à un anticléricalisme virulent. Comme dans le reste de l'Amérique latine, la transition vers une laïcité apaisée est récente. Elle est liée à l'état d'avancement du processus de démocratisation.

Mots-clés : Mexique, laïcité, Amérique latine, laïcisme, démocratisation.

\section{Abstract}

This text intends to show that Mexican secularism was built in a direct opposition to the Catholic Church. Although the position of the Church was decisive on a political and cultural level for a long time, it is now losing its social influence. Some features seem to be common to the different countries of the region, as the lack of clergy or the predominance of a more popular religiosity, and some characteristics look more peculiar to each national context, as the victory of the liberal forces, never questioned since the second half of the $19^{\text {th }}$ century. All of them contribute to the creation of a virulent prosecularism activism and anticlericalism. As in the rest of Latin America, the evolution towards a stable secularized regime is quite recent. It is linked to the steps achieved in the democratical transition.

Key words: Mexico, laicity, Latin America, democratization, laicism.

\section{Resumen}

En este texto pretendo mostrar que la historia de la laicidad mexicana se construye en relación a la presencia ineludible de una Iglesia Católica omnipresente en el tejido político y cultural, pero al mismo tiempo progresivamente debilitada en el ámbito social. Algunas particularidades de la región, como la falta de clero y el predominio de la religiosidad popular, aunadas a las particularidades nacionales, como el triunfo de las fuerzas liberales, jamás cuestionado desde mediados del siglo XIX, contribuyeron a la formación de un fuerte laicismo ligado a un anticlericalismo muy difundido. La transición hacia la laicidad, como en el resto de América Latina, es reciente, al mismo tiempo que se encuentra vinculada al proceso de democratización.

Palabras clave: México, laicidad, América Latina, laicismo, democratización. 